\title{
Fungistatic activity of all-trans retinoic acid against Aspergillus fumigatus and Candida albicans
}

This article was published in the following Dove Press journal:

Drug Design, Development and Therapy

29 April 2016

Number of times this article has been viewed

\author{
Elena Campione' \\ Roberta Gaziano² \\ Daniele Marino² \\ Augusto Orlandi ${ }^{3}$ \\ 'Department of Dermatology, \\ ${ }^{2}$ Department of Microbiology, \\ ${ }^{3}$ Department of Anatomic Pathology, \\ University of Rome Tor Vergata, Rome, \\ Italy
}

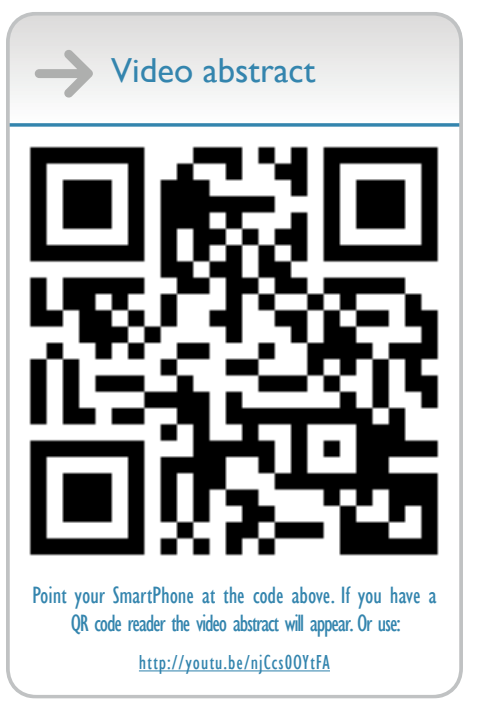

Correspondence: Elena Campione Department of Dermatology, University of Rome Tor Vergata, Viale Oxford 8I, 00133 Rome, Italy

Email campioneelena@hotmail.com
Purpose: Fungal infections are a major complication in hematologic and neoplastic patients causing severe morbidity and mortality. Aspergillus fumigatus and Candida albicans are among the most invasive opportunistic pathogens in immunocompromised patients, and classic antifungal drugs are frequently unsuccessful in these patients. Recent reports hypothesize that the antifungal efficacy of all-trans retinoic acid (ATRA) is mainly related to its strong capacity to stimulate monocyte-mediated immunity, but no consideration was given to its potential direct fungistatic activity. Moreover, ATRA offers the opportunity for systemic therapy.

Methods and results: We investigated the efficacy of ATRA at different concentrations for its antifungal activity against opportunistic $A$. fumigatus and $C$. albicans obtained from clinical samples according to standard protocols. A fungistatic activity of ATRA on A. fumigatus and C. albicans at $0.5-1 \mathrm{mM}$ concentration was documented up to 7 days.

Conclusion: This is the first evidence of a direct and strong fungistatic activity of ATRA against A. fumigatus and C. albicans. The potential adjuvant therapeutic application of ATRA might be useful in the treatment and/or prevention of systemic mycoses in immunocompromised patients. The discovery of a direct fungistatic activity, in association with its reported immunomodulatory properties, makes ATRA an excellent candidate for new combined antifungal strategies for systemic mycoses in immunocompromised and cancer patients.

Keywords: all-trans retinoic acid, fungistatic activity, fungal infections

\section{Introduction}

Fungal infections are a major complication in hematologic and neoplastic patients undergoing prolonged chemotherapy and often cause severe morbidity and mortality. In humans, Aspergillus fumigatus and Candida albicans are important fungal pathogens frequently causing severe infections with invasive growth in immunocompromised patients. ${ }^{1}$ Unfortunately, the use of classic antifungal drugs is unsuccessful in many of these patients. Retinoic acid - the biologically active metabolite of vitamin A - controls the normal immune system development as a modulator of both innate and adaptive immune responses. ${ }^{2}$ Vitamin $\mathrm{A}$ is a nutrient obtained through the diet either as provitamin-A (carotenoids) or as preformed vitamin A (retinol and retinyl esters). After its transport through the cytoplasmic binding with specific receptors, liver dehydrogenases convert retinol into retinoic acid, its biologically active metabolite..$^{3-7}$ Over the last decades, an increasing effort has been devoted to better define the involvement in the regulation of immune response, since vitamin A deficiency has been associated with an increased susceptibility to severe infectious diseases. ${ }^{8,9}$ The adding of all-trans retinoic acid (ATRA) to the therapy of acute promyelocytic leukemia resulted in a lower incidence of total episodes of fungemias in the patients. ${ }^{10}$ Recent studies support the hypothesis that efficacy of vitamin A or ATRA alone or in combination with other (c)
hereby accept the Terms. Non-commercial uses of the work are permitted without any further permission from Dove Medical Press Limited, provided the work is properly attributed. For permission hereby accept the Terms. Non-commercial uses of the work are permitted without any further permission from Dove Med 
drugs is related to its capacity to stimulate both innate and the adaptive immunity, in particular the monocyte-mediated immune response. ${ }^{4,10-12}$ We recently reported that tazarotene, a retinoic acid derivative, inhibits Tricophyton and C. albicans growth in vitro and a topical treatment with $0.1 \%$ tazarotene gel is effective in lateral onychomycosis. ${ }^{13}$ Since the contribution of inflammatory response is quite limited in the affected nail, we hypothesized that the beneficial effects of retinoids derive from a direct fungistatic activity. In comparison with tazarotene, ATRA offers the advantage of systemic therapy and is widely used in many patients safely. We aimed to investigate the efficacy of ATRA against opportunistic fungal pathogens such as $A$. fumigatus and $C$. albicans.

\section{Methods}

\section{Fungal culture conditions}

A. fumigatus and C. albicans strains used in this study were obtained from a clinical sample. The fungal strains were grown on Sabouraud dextrose agar (Difco Laboratories, Detroit, MI, USA) supplemented with chloramphenicol. Resting conidia of Aspergillus and Candida yeasts were harvested by washing the slant cultures with sterile saline. Swollen conidia were obtained by shaking at $300 \mathrm{rpm}$ at $37^{\circ} \mathrm{C}$ for 8 hours in Roswell Park Memorial Institute medium (RPMI) 1640 with $10 \%$ of fetal calf serum (FCS), until the spores swelled to almost twice their resting diameter. For generation of hyphae, swollen conidia were allowed to germinate $(98 \%$ germination) by further incubation in RPMI 1640 with $10 \%$ FCS ( $\sim 6$ hours). For the generation of hyphae, C. albicans yeasts were allowed to germinate ( $98 \%$ germination) by incubation at $37^{\circ} \mathrm{C}$ for 4 hours in RPMI 1640, $\mathrm{pH} 6.8-7.2$, with $10 \%$ FCS. To assess the effect of ATRA on the germination of $A$. fumigatus or $C$. albicans, resting conidia or yeast cells were plated on a 24 -well microplate at a density of $1 \times 10^{5}$ cells in $1 \mathrm{~mL}$ of RPMI 1640 with $10 \%$ FCS and supplemented with penicillin $(100 \mathrm{U} / \mathrm{mL})$ and streptomycin (100 $\mu \mathrm{g} / \mathrm{mL})$. ATRA (Sigma-Aldrich, St Louis, MO, USA) was added to the fungal suspension culture at time 0 at different concentrations (1, 0.5, 0.25, 0.12, and $0.06 \mathrm{mM})$. Fungal cultures were maintained at $37^{\circ} \mathrm{C}$ under continuous shaking at $300 \mathrm{rpm}$ and monitored every day until 1 week following treatment. The vitality of $C$. albicans yeasts and A. fumigatus conidia was evaluated by light microscopy using the trypan blue dye exclusion method. To confirm vitality after exposure to higher doses of ATRA for 7 days, conidia and yeast cells were harvested, washed, and again cultured with fresh medium containing $10 \% \mathrm{FCS}$, in the absence of ATRA. Under this condition, conidia and yeasts were able to germinate into hyphae within 24 hours.

\section{Evaluation of fungistatic activity}

The development of $A$. fumigatus and C. albicans was followed using an optical microscope (Carl Zeiss Meditec AG, Jena, Germany) with a $40 \times$ magnification objective lens. Microscopic images were recorded after 8 and 4 hours of incubation with ATRA at $37^{\circ} \mathrm{C}$ for $A$. fumigatus conidial swelling and $C$. albicans yeast cell germination, respectively.

\section{Results}

Light optical microscopy revealed that, at the earliest time point ( 8 hours) in the presence of ATRA ( $1 \mathrm{mM})$, A. fumigatus conidia did not swell and remained similar in shape and size to the resting ones (Figure 1B). On the contrary, as shown in Figure 1A, most of the control untreated conidia ( $~ 80 \%)$ were swollen and some developed short germ tube similar to those observed in the ATRA-treated cultures of nonsynchronized population of $A$. fumigatus at doses $<1 \mathrm{mM}$ (Figure 1C). After more prolonged incubation (24 hours), all control and ATRA-treated conidia at doses $<1 \mathrm{mM}$ were swollen and produced a germ tube (data not shown). ATRA at 1 and $0.5 \mathrm{mM}$ also inhibited germination and hyphal outgrowth of C. albicans (Figure 1E and F). Instead, control untreated yeast cells developed germ tube and subsequently hyphal growth (Figure 1D). Doses of ATRA $<0.5 \mathrm{mM}$ did not affect the ability of C. albicans to germinate (Figure $1 \mathrm{G}$ ). The inhibitory effect of ATRA on the germination of A. fumigatus and C. albicans was maintained and examined at a later time point (day 7). These data suggested that the fungistatic activity of ATRA at $0.5-1 \mathrm{mM}$ concentration was obtained without any adverse effects on A. fumigatus conidia and $C$. albicans yeast, since their viability was $>90 \%$ as assessed by trypan blue. Similar results were observed at other time points (data not shown). To confirm the vitality of $A$. fumigatus and $C$. albicans after exposure to higher $(0.5-1 \mathrm{mM})$ doses, conidia and yeast cells were harvested, washed after 7 days, and again cultured in the absence of ATRA. A. fumigatus conidia and C. albicans yeasts were able to germinate into hyphae within 24 hours (data not shown), again supporting the fungistatic activity of ATRA in vitro.

\section{Discussion}

Our results documented the fungistatic activity of ATRA at 0.5-1 mM concentration on A. fumigatus and C. albicans. 

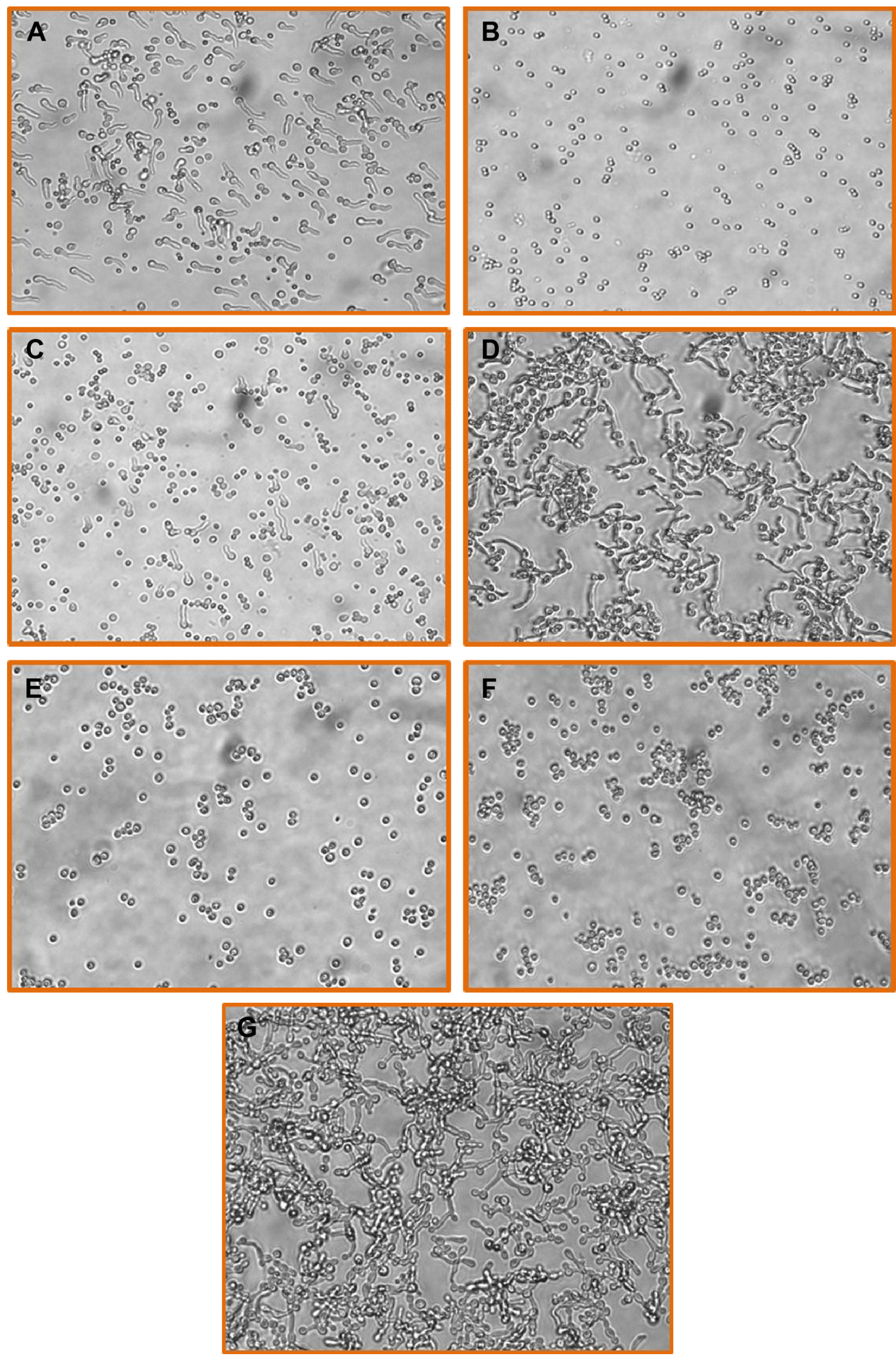

Figure I Inhibitory effect of ATRA on Asperillus and Candida germination.

Notes: Resting Aspergillus fumigatus conidia or Candida albicans yeast cells were cultured in RPMI 1640 with $10 \% \mathrm{FCS}$ at $37^{\circ} \mathrm{C}$ in the presence or absence of ATRA.

(A) Conidia untreated, (B) conidia treated with ATRA I mM, (C) conidia treated with ATRA 0.5 mM, (D) Candida yeasts untreated, (E) Candida yeasts treated with ATRA I mM, (F) Candida yeasts treated with ATRA $0.5 \mathrm{mM}$, and (G) Candida yeasts treated with ATRA $0.25 \mathrm{mM}$. The germination of both fungi was followed using an optical microscope. Microscopic images were recorded after 8 and 4 hours of incubation for $A$. fumigatus conidia and $C$. albicans yeasts, respectively. Magnification: $\times 40$.

Abbreviations: FCS, fetal calf serum; ATRA, all-trans retinoic acid. 
ATRA offers the opportunity for systemic therapy against opportunistic fungal pathogens such as A. fumigatus and C. albicans. Despite an increasing interest in the immunomodulatory role of ATRA, its specific role in the immune response to fungal infections was never explored. Our study is the first evidence of a direct and high fungistatic activity of ATRA against A. fumigatus and C. albicans. Till now, several in vitro studies highlighted the function of vitamin A as an important factor for immune system development and a modulator of the innate and the adaptive immune response. ${ }^{6-9}$ As a matter of fact, vitamin A regulates the development of B lymphocytes and immunoglobulin production. ${ }^{14}$ Retinoic acid also shows specific immunomodulatory activities. In T lymphocytes, retinoic acid attenuated the Th1-associated gene expression and skewed the immune response toward a Th2 profile. ${ }^{15}$ Retinoic acid also modulated the production of LPS-induced cytokines and/or chemokines in monocytes, macrophages, and dendritic cells. ${ }^{16,17}$ Moreover, ATRA induced proliferative arrest in nonmyeloid mesenchymal cells. ${ }^{18,19}$ ATRA has been shown to stimulate the differentiation of myeloid-derived suppressor cells to dendritic cells and macrophages. At therapeutic concentrations, ATRA could substantially drop the number of myeloid-derived suppressor cells in tumor-bearing mice and in patients with cancer, thus improving their antigen-specific response. ${ }^{20,21}$ Lei et $\mathrm{al}^{11}$ hypothesized that ATRA treatment, alone and in combination with an 8-aminoquinoline primaquine, can push lung myeloid-derived suppressor cells to differentiate in functional macrophages helping to clear pneumonia from the Pneumocystis carinii infection. Klassert et al ${ }^{12}$ recently reported the effects of ATRA on the immune response of human monocytes during $C$. albicans infection. The authors documented an immunomodulatory effect of ATRA leading to a highly significant suppression of the fungi-induced expression of TNF $\alpha$, IL6, and IL12 at both the transcriptional and posttranslational levels. ${ }^{12}$

The effectiveness of ATRA and its possible toxicity can be easily monitored in the blood. Our results in vitro are already widely proven in the clinical practice since patients affected by promyelocytic leukemia were treated with ATRA, they were less subjected to systemic infections, with a more preserved immunological profile. ${ }^{10}$ The adjuvant application of ATRA might also be useful in the treatment and/ or prevention of systemic mycoses in immunocompromised patients. Therefore, the discovery of a direct fungistatic activity associated with its reported immunomodulatory properties makes ATRA an excellent candidate for new and efficient antifungal strategies. ATRA may be administered alone or in combination with lower doses of conventional antimycotic agents, thus reducing their side effects. Although the therapeutic systemic use of ATRA as an adjuvant for antifungal therapies requires further validation in preclinical models, the considerable health burden represented by invasive mycosis and the emerging multidrug resistance justifies every effort directed to the development of new therapeutic solutions.

\section{Acknowledgment}

The authors wish to thank Denis Mariano for editing assistance. In memory of Professor Chimenti who recently passed away.

\section{Disclosure}

The authors report no conflicts of interest in this work.

\section{References}

1. Pfaller MA, Diekema DJ. Epidemiology of invasive candidiasis: a persistent public health problem. Clin Microbiol Rev. 2007;20(1):133-163.

2. Majer O, Bourgeois C, Zwolanek F, et al. Type I interferons promote fatal immunopathology by regulating inflammatory monocytes and neutrophils during Candida infections. PLoS Pathog. 2012;8(7):e1002811.

3. D'Ambrosio DN, Clugston RD, Blaner WS. Vitamin A metabolism: an update. Nutrients. 2011;3(1):63-103.

4. Wolf G. A history of vitamin A and retinoids. FASEB J. 1996;10(9): 1102-1107.

5. Doldo E, Costanza G, Agostinelli S, et al. Vitamin A, cancer treatment and prevention: the new role of cellular retinol binding proteins. Biomed Res Int. 2015;2015:624627.

6. Hall JA, Grainger JR, Spencer SP, Belkaid Y. The role of retinoic acid in tolerance and immunity. Immunity. 2011;35(1):13-22.

7. Theodosiou M, Laudet V, Schubert M. From carrot to clinic: an overview of the retinoic acid signaling pathway. Cell Mol Life Sci. 2010; 67(9):1423-1445.

8. Clagett-Dame M, Knutson D. Vitamin A in reproduction and development. Nutrients. 2011;3(4):385-428.

9. Mark M, Ghyselinck NB, Chambon P. Function of retinoic acid receptors during embryonic development. Nucl Recept Signal. 2009;7:e02.

10. Girmenia C, Lo Coco F, Breccia M, et al. Infectious complications in patients with acute promyelocytic leukaemia treated with the AIDA regimen. Leukemia. 2003;17(5):925-930.

11. Lei GS, Zhang C, Shao S, Jung HW, Durant PJ, Lee CH. All-transretinoic acid in combination with primaquine clears pneumocystis infection. PLoS One. 2013;8(1):e53479.

12. Klassert TE, Hanisch A, Bräuer J, et al. Modulatory role of vitamin A on the Candida albicans-induced immune response in human monocytes. Med Microbiol Immunol. 2014;203(6):415-424.

13. Campione E, Paternò EJ, Costanza G, et al. Tazarotene as alternative topical treatment for onychomycosis. Drug Des Devel Ther. 2015;9: 879-886.

14. Sommer A, Vyas KS. A global clinical view on vitamin A and carotenoids. Am J Clin Nutr. 2012;96(5):1204S-1206S.

15. Dawson HD, Collins G, Pyle R, et al. Direct and indirect effects of retinoic acid on human Th2 cytokine and chemokine expression by human T lymphocytes. BMC Immunol. 2006;7:27.

16. Tsai YC, Chang HW, Chang TT, et al. Effects of all-trans retinoic acid on Th1-and Th2-related chemokines production in monocytes. Inflammation. 2008;31(6):428-433.

17. Wojtal KA, Wolfram L, Frey-Wagner I, et al. The effects of vitamin A on cells of innate immunity in vitro. Toxicol In Vitro. 2013;27(5): $1525-1532$. 
18. Orlandi A, Francesconi A, Cocchia D, et al. Phenotypic heterogeneity influences apoptotic susceptibility to retinoic acid and cis-platinum of rat arterial smooth muscle cells in vitro: Implications for the evolution of experimental intimal thickening. Arterioscler Thromb Vasc Biol. 2001;21(7):1118-1123.

19. Orlandi A, Pucci S, Ciucci A, Pichiorri F, et al. Modulation of clusterin isoforms is associated with all-trans retinoic acid-induced proliferative arrest and apoptosis of intimal smooth muscle cells. Arterioscler Thromb Vasc Biol. 2005;25(2):348-353.
20. Nefedova Y, Fishman M, Sherman S, et al. Mechanism of all-trans retinoic acid effect on tumor-associated myeloid-derived suppressor cells. Cancer Res. 2007;67(22):11021-11028.

21. Lee JM, Seo JH, Kim YJ, et al. The restoration of myeloid-derived suppressor cells as functional antigen-presenting cells by NKT cell help and all-trans-retinoic acid treatment. Int J Cancer. 2012;131(3):741-751.

\section{Publish your work in this journal}

Drug Design, Development and Therapy is an international, peerreviewed open-access journal that spans the spectrum of drug design and development through to clinical applications. Clinical outcomes, patient safety, and programs for the development and effective, safe, and sustained use of medicines are a feature of the journal, which has also been accepted for indexing on PubMed Central. The manuscript management system is completely online and includes a very quick and fair peer-review system, which is all easy to use. Visit http://www.dovepress.com/testimonials.php to read real quotes from published authors.

Submit your manuscript here: http://www.dovepress.com/drug-design-development-and-therapy-journal 